\title{
Recidiva con el tratamiento combinado (infiltración intralesional con betametasona, resección quirúrgica y radioterapia) en cicatrices queloides en pabellón auricular
}

\author{
Recurrence with combined treatment (intralesional infiltration \\ with betamethasone, surgical resection and radiotherapy) \\ in keloid scars in the atrial pavilion \\ Dra. Claudia Gutiérrez-Gómez,* Dr. Joseph Bañuelos-Mancilla, \\ Dra. Clarisa Esther Del Hierro-Gutiérrez, ${ }^{\ddagger}$ Dra. Brenda Valdez-Díaz ${ }^{\S}$
}

Palabras clave: Cicatriz queloide, infiltración intralesional, radioterapia.

Keywords: Keloid scar, intralesional infiltration, radiotherapy.

* Cirujana plástica comisionada al

Laboratorio de Biotecnología CENIAQ, Instituto Nacional de

Rehabilitación «Dr. Luis Guillermo Ibarra Ibarra». Hospital General «Dr.

Manuel Gea González».

* Médico pasante

en Servicio Social, Universidad «La Salle».

$\S$ Médico pasante

en Servicio Social, Universidad Nacional

Autónoma de México.

Los autores de este artículo no tienen conflicto de intereses qué declarar.

Recibido:

23 enero 2019 Aceptado para publicar: 14 marzo 2019

\section{RESUMEN}

La cicatrización queloide es un problema clínico de difícil manejo en el que el tratamiento combinado puede disminuir su recidiva hasta llegar a representar de 4 a $27 \%$. El objetivo de este estudio fue identificar la recidiva con el tratamiento combinado de infiltración intralesional de betametasona, resección quirúrgica y radioterapia en el tratamiento de cicatrices queloides en el pabellón auricular. El diseño del estudio fue observacional descriptivo, transversal y retrospectivo. Analizamos los expedientes clínicos de pacientes del Hospital General «Dr. Manuel Gea González» que presentaban cicatrices queloides en pabellón auricular y recibieron tratamiento combinado en un lapso de cinco años. Veintiún pacientes cumplieron con los criterios para ser incluidos en el estudio; el $81.3 \%$ tenía entre 16 y 30 años de edad; recibieron de tres a seis sesiones de radioterapia, con una media de cuatro. El $80.9 \%$ se encontraban en el lóbulo y se reportó recidiva en $9.5 \%$. El seguimiento de los pacientes fue de 12 a 60 meses. En 2012 reportamos un protocolo de estudio del manejo de cicatrices queloides en el pabellón auricular en 51 pacientes con una recurrencia de $12.5 \%$ con la utilización de infiltración de betametasona, resección quirúrgica y colchicina. Concluimos que el tratamiento combinado presenta una recidiva de $9.5 \%$ en nuestra población de estudio y fue una limitante el número de casos.

\section{ABSTRACT}

Keloid scarring is a difficult-to-handle clinical problem in which a combined treatment can decrease its recurrence until it reaches 4 to $27 \%$. The objective of this study was to identify recurrence with the combined treatment of intralesional infiltration of betamethasone, surgical resection and radiotherapy in the treatment of keloid scars in the atrial pavilion. The study design was observational descriptive, cross-sectional and retrospective. We analyzed the clinical records of patients from the «Dr. Manuel Gea González» General Hospital who presented keloid scars in the atrial pavilion and received combined treatment within a period of five years. Twenty-one patients met the criteria to be included in the study; $81.3 \%$ were between 16 and 30 years old. They received three to six radiotherapy sessions, with an average of four. In $80.9 \%$ they were in the lobe and recurrence was reported in $9.5 \%$. The follow-up of the patients was 12 to 60 months. In 2012 we reported a study protocol for the handling of keloid scars in the atrial pavilion in 51 patients with a recurrence of $12.5 \%$ with the use of betamethasone infiltration, surgical resection and colchicine. We conclude that the combined treatment presents a recurrence of $9.5 \%$ in our study population and the number of cases was a limitation.

\section{INTRODUCCIÓN}

T a cicatriz queloide es uno de los grandes $\amalg$ retos para el cirujano plástico, puesto que se trata de uno de los problemas clínicos más frustrantes dentro de la cicatrización anormal. Las cicatrices queloides se originan como consecuencia de una lesión dérmica y exhiben un crecimiento exuberante e indefinido de colágena. Existe una propensión que se presenten 
en individuos de piel oscura, con tendencia familiar y rara vez en los extremos de la vida. Se atribuyen a una alteración en la regulación de los factores de crecimiento, recambio aberrante de colágena, factores genéticos, disfunción inmunológica, reacción al sebo y alteraciones mecánicas, por lo que no hay una hipótesis única que explique el proceso adecuadamente. Las múltiples modalidades terapéuticas existentes, incluidas la resección quirúrgica, la infiltración de esteroides, radioterapia, láser, bleomicina, 5 fluorouracilo, crioterapia, uso de silicona y de presoterapia, entre otras, hablan del poco conocimiento que tenemos de esta patología.

La primera descripción escrita se atribuye a los egipcios y aparece en los papiros de Edwin Smith. En 1806 Alibert acuñó el término queloide, procedente de la terminología griega «pinza de cangrejo». Cosman y colaboradores documentaron la presentación, características y tratamiento de los queloides en la primera revisión sistemática al respecto realizada en 1961. Posteriormente Mancini, Quaife y Peacock delinearon las diferencias clínicas entre queloides y cicatrices hipertróficas. ${ }^{1-3}$

Entre las hipótesis planteadas para explicar la formación y crecimiento de las queloides están el aumento en la actividad del factor de crecimiento (factor de crecimiento transformante beta y factor de crecimiento derivado de las plaquetas), alteración en la matriz extracelular (aumento de fibronectina, producción aberrante de proteoglicanos y disminución en los niveles de ácido hialurónico) y regulación anormal del equilibrio de la colágena (fibras de colágena más gruesas, onduladas y existencia de nódulos). Tanto las cicatrices queloides infiltradas con esteroides como las radiadas presentan una disminución en los inhibidores de colágenas y un incremento en la apoptosis de los fibroblastos, lo que lleva a una normalización de los niveles de la red de colágena.

Tensión mecánica. La tensión mecánica en una cicatriz desalinea la orientación de las fibras de colágena y provoca una cicatriz queloide. Las cicatrices anormales rara vez se presentan en pacientes mayores cuya piel ya no tiene tensión; sin embargo, los dos sitios más frecuentes de queloides son el tórax y el lóbulo auricular, cuyas fuerzas tensiles no son similares. La fuerza y tensión se postulan como factores importantes en la apariencia final de la cicatrización; no obstante, es posible que jueguen un papel más importante en la patogénesis de las cicatrices hipertróficas que en la formación de queloides. ${ }^{1}$

Disfunción inmunológica genética. Algunos estudios sugieren la asociación del grupo sanguíneo $A$, así como antígenos leucocitarios humanos B14, BW35, DR5 y DQW3 en pacientes con queloides. Múltiples informes señalan alteraciones en el comportamiento sérico y las inmunoglobulinas $\mathrm{G}$ y $\mathrm{M}$ en pacientes con queloides. La evidencia clínica sugiere que aquéllos que desarrollan queloides tienen una hipersensibilidad inherente del sistema inmunológico mediado por células, como reacción inmunológica al sebo. La lesión dérmica expone la unidad pilosebácea a la circulación sistémica, iniciando así una respuesta mediada por células en los individuos que tienen linfocitos $\mathrm{T}$ sensibles al sebo.

En el servicio de cirugía plástica cambió en los últimos años el protocolo por la disponibilidad del uso de la radioterapia como manejo adyuvante, por lo que consideramos importante conocer el porcentaje de recidiva con este manejo.

El objetivo del estudio fue conocer la recidiva con el tratamiento combinado de infiltración intralesional con betametasona (de lo que se dispone en nuestro país, ya que no se vende triamcinolona en forma comercial), resección quirúrgica y radioterapia.

\section{MATERIAL Y MÉTODO}

Se realizó un estudio observacional descriptivo, retrospectivo y transversal en el Servicio de Cirugía Plástica del Hospital General «Dr. Manuel Gea González» en un periodo de cinco años, utilizando la base de datos de pacientes con cicatriz queloide en el pabellón auricular. Se incluyeron los expedientes clínicos aquéllos que tenían cicatrices queloides en pabellón auricular y recibieron terapia combinada con infiltración con betametasona, resección quirúrgica y radioterapia. Se trataba de pacientes de cualquier edad y ambos sexos; se excluyeron expedientes de pacientes cuyo seguimiento hubiera sido menor a 12 meses y se eliminaron los casos cuyos expedientes estuvieran incompletos. 
Las variables a considerar fueron: edad, sexo, lateralidad (derecho o izquierdo), área anatómica del pabellón auricular, estudio histopatológico confirmatorio de diagnóstico, etiología, número de sesiones, infiltración de Diprospan $1 \mathrm{~mL}$ (Schering Plough) (equivalente a dipropionato de betametasona equivalente a $5 \mathrm{mg}$, fosfato sódico de betametasona equivalente a $2 \mathrm{mg}$ de betametasona): aplicación de $1 \mathrm{~mL}$ de betametasona intralesional en forma preoperatoria en tres ocasiones con intervalo de seis semanas y una cuarta dosis el día de la cirugía después de la resección en forma transoperatoria. La resección quirúrgica se realizó con anestesia local, ya sea con resección en huso o mediante fileteado de la piel para un cierre sin tensión en lesiones mayores a $2 \mathrm{~cm}$. Las sesiones de radioterapia fueron establecidas por el Servicio de Radioterapia del Instituto Nacional de Cancerología de acuerdo a protocolos establecidos para partes blandas.

\section{RESULTADOS}

De 95 expedientes revisados, sólo 21 cumplieron con los criterios para ser incluidos en el estudio. Seis casos fueron del sexo masculino (29\%) y 15 del sexo femenino (71\%). El rango de edad fue de 10 a 61 años y $81.3 \%$ de los pacientes se encontraba entre 16 y 30 años. En cuanto a la localización, 17 se ubicaban en el lóbulo (80.9\%), dos en hélix $(9.5 \%)$ y dos retroauriculares $(9.5 \%)$.

En cuanto a la etiología, 18 casos (85.7\%) fueron secundarios a perforación, dos casos a postcirugía $(9.5 \%)$ y un caso $(4.7 \%)$ por mordedura. En cuanto a lateralidad, 14 casos (66.6\%) fueron del lado derecho y siete (33.3\%) del izquierdo.

El tratamiento con radioterapia externa de baja energía en la herida quirúrgica con un margen de $4 \mathrm{~mm}$ se dio entre tres y seis sesiones, con una media de cuatro por paciente. La dosis fue entre 12 y 20 unidades Gray (Gy), con una media de 15.6 Gy (Tabla 1).

Se reportó recidiva en dos pacientes (9.5\%). En un caso la recidiva cedió con radioterapia con un seguimiento a 12 meses. El segundo caso respondió a infiltración intralesional de betametasona con seguimiento a 16 meses.

El seguimiento de los pacientes fue entre 12 y 60 meses.

\begin{tabular}{|c|c|c|}
\hline Caso & No. sesiones & $\begin{array}{c}\text { Dosis } \\
\text { Unidades } \\
\text { Gray (Gy) }\end{array}$ \\
\hline 1 & 3 & 15 \\
\hline 2 & 3 & 15 \\
\hline 3 & 3 & 15 \\
\hline 4 & 6 & 18 \\
\hline 5 & 5 & 20 \\
\hline 6 & 5 & 20 \\
\hline 7 & 6 & 18 \\
\hline 8 & 4 & 16 \\
\hline 9 & 3 & 15 \\
\hline 10 & 4 & 16 \\
\hline 11 & 5 & 15 \\
\hline 12 & 3 & 12 \\
\hline 13 & 3 & 15 \\
\hline 14 & 6 & 18 \\
\hline 15 & 3 & 15 \\
\hline 16 & 4 & 16 \\
\hline 17 & 4 & 16 \\
\hline 18 & 3 & 12 \\
\hline 19 & 3 & 15 \\
\hline 20 & 3 & 15 \\
\hline 21 & 3 & 12 \\
\hline Total & 82 & 329 \\
\hline
\end{tabular}

\section{DISCUSIÓN}

Es bien sabido el efecto benéfico de la terapia combinada en el tratamiento de cicatrices queloides que logra disminuir las recidivas hasta en $10 \%$. Los trabajos que promueven la monoterapia en el tratamiento de las cicatrices queloides son escasos, pero además sin un seguimiento a largo plazo en el que efectivamente se reduzca la recidiva al $0 \%{ }^{4}$ Algunos autores enfatizan sobre el hecho de cerrar una herida sin tensión después de resecar un queloide y el emplear la técnica del fileteado que ayuda a disminuir la recidiva; ${ }^{5} \sin$ embargo, la historia nos muestra que la mayoría de las veces es más prudente emplear un tratamiento combinado. En publicaciones previas, Gutiérrez y colegas ${ }^{6}$ presentaron un caso de muy difícil manejo, con tres cirugías previas y recidiva severa, caso que se trató con 
radioterapia, logrando un control postoperatorio libre de tumor de más de 10 años.

La colchicina incrementa la actividad de la colagenasa y ha sido recomendada por Peacock y Gutiérrez. ${ }^{6,7}$ Por otra parte, Bisbal ${ }^{8}$ señala una recidiva de $4 \%$ con la utilización de cirugía seguida de la aplicación de braquiterapia y radioterapia externa. Kovalic, Escarmant y Ollstein reportan recidivas de 21 a $27 \%$ en seguimientos a largo plazo. ${ }^{9-12}$

En 2012, Gutiérrez ${ }^{13}$ reportó un estudio de protocolo de manejo de cicatrices queloides en el pabellón auricular en 51 pacientes; 18.6\% de las cicatrices tratadas cedieron con la infiltración intralesional. De las 48 cicatrices que recibieron tratamiento quirúrgico hubo recidiva en seis $(12.5 \%)$, con un seguimiento entre ocho meses y seis años. ${ }^{13}$

Otras terapias utilizadas son la crioterapia intralesional, ya sea mediante un dispositivo desechable basado en la administración de nitrógeno líquido o mediante un dispositivo basado en gas Argón. Reportan recidivas a un año de hasta el $17 \%$, con la ventaja de disminuir la sintomatología, pero con hiperpigmentación residual. ${ }^{14,15}$ Payapvipapong ${ }^{16}$ reporta que no encontró diferencia significativa entre la infiltración de triamcinolona o bleomicina intralesional. Asimismo, Khan ${ }^{17}$ reportó un mejor resultado y menos complicaciones con la infiltración combinada de triamcinolona y 5 fluorouracilo.

\section{CONCLUSIONES}

El tratamiento combinado en las cicatrices queloides de las orejas con infiltración de betametasona, resección quirúrgica y radioterapia presenta una recidiva de $9.5 \%$ en nuestra población de estudio, con una disminución de $3 \%$ con respecto al grupo previo en quienes no se utilizó radioterapia. La limitante del estudio fue el número de casos.

\section{REFERENCIAS}

1. Al-Attar A, Mess S, Tomasen JM, Kauffman CL, Davison SP. Keloid pathogenesis and treatment. Plast Reconstr Surg 2006; 117 (1): 286.

2. Breasted JH. The Edwin Smith surgical papyrus, Vol. 1 (hieroglyphic translation and commentary). Chicago: University of Chicago Press, 1930, pp. 403-406.

3. Mustoe TA, Cooter RD, Gold MH, Hobbs FD, Ramelet AA, Shakespeare PG et al. International clinical recommendations on scar management. Plast Reconstr Surg 2002; 110: 560.

4. Cuenca-Pardo J, Hernández Valverde CB, Alvarez-Díaz C, Luján-Olivar FS, Escalona-Mancilla A. Tratamiento quirúrgico de cicatrices queloides en oreja. Cir Plast Iberolatinoam 2006; 16 (2): 78.

5. Kim DY, Kim ES, Eo SR, Kim KS, Lee SY, Cho BH. A surgical approach for earlobe keloid: keloid fillet flap. Plast Reconstr Surg 2004; 113 (6): 1668.

6. Gutiérrez C, Guinto P, Andrade L, Alfaro HJ. Uso de la colchicina como adyuvante en el tratamiento de cicatrices queloides recidivantes. Reporte de un caso. Cir Plast Iberolatinoam 2001; 11 (3): 126.

7. Peacock EE Jr. Pharmacologic control of surface scarring in human beings. Ann Surg 1981; 193: 592.

8. Bisbal J, Guix B, Coronel R. Tratamiento combinado de los queloides mediante cirugía y braquiterapia. Cir Plast Iberolatinoam 2009; 35 (4): 283.

9. Burd A, Huang L. Hypertrophic response and keloid diathesis: two very different forms of scar. Plast Reconstr Surg 2005; 116 (7): 150.

10. Kovalic JJ, Perez CA. Radiation therapy following keloidectomy: A 20 year experience. Int J Radiat Oncol Biol Phys 1989; 17 (1): 77.

11. Escarmant P, Zimmermann S, Amar A, Ratoanina JL, Moris A, Azaloux H et al. The treatment of 783 keloid scars by iridum 192 intersticial irradiation after surgical excision. Int J Radiat Oncol Biol Phys 1993; 26 (2): 245.

12. Ollstein RN, Sieguel HW, Gillooley J, Barsa JM. Treatment of keloids by combined surgical excision and immediate post-operative X-ray therapy. Ann Plast Surg 1981; 7 (4): 281-285.

13. Gutiérrez-Gómez C, López-Mendoza FJ, LaraOntiveros J, Cervantes-Díaz JP, Márquez-Gutiérrez E, Morales-Naya D. Protocolo de tratamiento de cicatrices queloides en el pabellón auricular del Hospital General Dr. Manuel Gea González. Cir Plast Iberolatinoam 2012; 30 (1): 49-54.

14. Van Leeuwen MC, Van der Wal MB, Bulstra AE, GalindoGarre F, Molier J, Van Zuijlen PP et al. Intralesional cryotherapy for treatment of keloid scars: a prospective study. Plast Reconstr Surg 2015; 135 (2): 580-589.

15. Van Leeuwen MC, Bulstra AE, Van Leewen PA, Niesses FB. A new argon gas-based device for the treatment of keloid scars with the use of intralesional cryotherapy. J Past Reconstr Aesthet Surg 2014; 67 (12): 1703-1710.

16. Payapvipapong K, Niumpradit N, Piriyanand C, Buranaphalin S, Nakakes A. The treatment of keloids and hypertrophic scars with intralesional bleomycin in skin of color. J Cosmet Dermatol 2015; 14 (1): 83-90.

17. Khan MA, BashirMM, Khan FA. Intralesional triamcinolone alone and in combination with 5-fluorouracil for the treatment of keloid and hypertrophic scars. J Pak Med Assoc 2014; 64 (9): 1003-1007.

\section{Correspondencia:}

Dra. Claudia Gutiérrez Gómez

Hospital Médica Sur

Puente de Piedra Núm. 150, Torre 2,

Consultorio 420,

Col. Toriello Guerra, 14050,

Alcaldía Tlalpan, México, Ciudad de México.

E-mail:dra.claugg8@gmail.com 